\title{
Safety, efficacy, and patient acceptability of lidocaine hydrochloride ophthalmic gel as a topical ocular anesthetic for use in ophthalmic procedures
}

This article was published in the following Dove Press journal:

Clinical Ophthalmology

21 October 2009

Number of times this article has been viewed

\author{
Michael A Page \\ Frederick W Fraunfelder \\ Department of Ophthalmology \\ (Casey Eye Institute), Oregon Health \\ and Science University, Portland, \\ OR, USA
}

Purpose: To review the current literature on safety, efficacy, and measures of surgeon and patient satisfaction with lidocaine hydrochloride gel as a tool for ocular anesthesia.

Methods: Pubmed search using keywords "lidocaine gel," "ophthalmic," and "surgery" and compiling cross-references. Twenty-six total references were reviewed, including 15 prospective randomized controlled trials ( $\mathrm{RCTs}$, total $\mathrm{N}=933$, average $\mathrm{N}=62$ ), 6 nonrandomized prospective studies (total $\mathrm{N}=234$, average $\mathrm{N}=39$ ), 2 animal studies, 1 microbiologic study, and 2 letters to the editor.

Results: The RCTs and nonrandomized prospective studies evaluated a number of measures including timing of onset of anesthesia, duration of anesthesia, intraoperative and postoperative pain, need for additional anesthetic applications, intracameral lidocaine levels, and adverse effects. Control groups received topical drops, subconjunctival anesthetic, retrobulbar anesthetic, or sham gel. Lidocaine gel was shown to be at least as effective for pain control as alternative therapies in all studies, with longer duration of action than topical drops. Patient and surgeon satisfaction were high, and adverse effects were rare and comparable to those for anesthetic drop formulations. Surgical settings included cataract, pterygium, trabeculectomy, strabismus, intravitreal injection, vitrectomy, and penetrating keratoplasty.

Conclusions: Lidocaine gel is a safe, effective, and potentially underutilized tool for ophthalmic surgery.

Keywords: lidocaine, gel, topical, ophthalmic, ocular, anesthetic

\section{Introduction}

Local anesthesia for ophthalmic surgery was first introduced by Koller in $1884{ }^{1}$ With the help of newly discovered topical cocaine, ophthalmic procedures could be performed more skillfully and more safely than with the conventional adjuncts of general anesthesia, hypnosis, or no anesthesia at all. Within weeks of Koller's discovery of the topical efficacy of cocaine, other practitioners began to experiment with intraorbital injections of the drug. Surgeons soon realized that retrobulbar cocaine afforded unprecedented ability to produce total anesthesia and akinesia of the globe, and a wave of ophthalmic surgical advances ensued. ${ }^{2}$

Cocaine was lauded for its potent vasoconstrictive activity and its rapid blockade of sensory and motor nerves. The excitement over its retrobulbar use was tempered, however, by early reports of blindness, respiratory depression, and death with injection near the orbital apex. ${ }^{3}$

With the subsequent discovery of epinephrine and other pharmacologic advances, ophthalmic anesthesia became safer and more refined throughout the 20th century.
Correspondence: FW (Rick) Fraunfelder Casey Eye Institute Cornea Service, 3375 SW Terwilliger Blvd., Portland, OR $97239-4197$, USA

$\mathrm{Tel}+\mathrm{I} 5034947674$

Fax +I 5034182284

Email eyedrug@ohsu.edu 
In the 1930s, procaine and lidocaine were found to be cheaper and more stable than cocaine. In 1949, Atkinson reported that the addition of hyaluronidase could facilitate safe, long-lasting, large-volume retrobulbar nerve blockade. ${ }^{4}$ Variations on this technique continued to dominate anesthesia for intraocular surgery through the 1970s and 1980s.

The first cataract phacoemulsification in 1972 forecast a trend of faster, cleaner ophthalmic surgery through smaller and smaller incisions. As the tools improved and the paradigm shifted, many surgeons found themselves able to return to purely topical anesthetic techniques in order to mitigate the discomfort and risks associated with retrobulbar or peribulbar injection.

\section{Topical anesthetics}

Topical anesthetics work by reversibly blocking sodium channels and preventing propagation of painful nerve impulses in the cornea, conjunctiva, and sclera. In the cornea, nerve endings are superficial and protected only by the tear film and by a thin layer of stratified epithelium that is permeable to lipid- and aqueous-soluble molecules. Nerves in the conjunctiva are covered by nonkeratinized stratified epithelium, readily penetrated by topical anesthetics if $\mathrm{pH}$ conditions are optimal. ${ }^{5}$

Tetracaine is available in a non-preserved form and penetrates an intact tear film if formulated to match the $\mathrm{pH}$ (7.6 to 7.8) of the tear film. Lidocaine behaves in a similar fashion. Without $\mathrm{pH}$-adjusting additives, these anesthetics typically cause significant stinging on application and carry reduced patient acceptability in office or emergency department settings. Proparacaine and benoxinate contain the bacteriocide benzalkonium chloride, which acts to disrupt the lipid and mucous layers of the tear film and allow these anesthetics more direct access to corneal and conjunctival nerve endings via the unprotected epithelium. ${ }^{5}$

Topical anesthetic drops are convenient, typically safe, and provide rapid onset of anesthesia. Corneal anesthesia is especially rapid and complete. Drawbacks to topical drops include incomplete anesthesia of the conjunctiva and sclera with a single application, potential toxicity to the corneal epithelium, and the need for multiple drop applications over the course of a given ophthalmic procedure.

Gel formulations of topical anesthetics have been in use in the fields of urology and otolaryngology for many years; due to their viscous qualities they are favored for anesthesia of mucous membranes and other areas where high flow of saliva, urine, and secretions may dilute an aqueous anesthetic and reduce its effectiveness. It follows that aqueous anesthetics would be subject to the same diluent effect in the eye, with ongoing tear film turnover and the use of irrigants during ophthalmic surgery. ${ }^{3}$

Over the last 10 years a number of reports have been submitted on the utility of gel anesthetic, specifically lidocaine gel, for ocular procedures. The purpose of this paper is to review the literature on lidocaine gel in ophthalmic surgery, and comment on overall safety, efficacy, and patient acceptability of this potentially underutilized surgical tool.

\section{Methods}

A Pubmed search was conducted using the terms "lidocaine gel" and "ophthalmic" and restricted to English-language studies. There was no restriction on year of publication. Twenty-six listings were initially returned, and bibliographic references from each study were explored and added to the review. Articles on pharmacokinetics, basic physiology, and basic pharmacology were omitted from the review. A total of 25 clinically relevant studies were included for analysis and discussion.

\section{Results}

Of the 25 relevant studies, 15 were prospective randomized controlled trials. Six were nonrandomized prospective studies, and 1 was a large retrospective case series. Two animal studies and 1 microbiologic study were included. Two letters to the editor are also referenced.

The prospective studies on lidocaine gel in ophthalmic surgery covered a wide variety of operative situations and there were relevant data to review for each of the ophthalmic surgical subspecialties. Eight studies addressed the use of lidocaine gel in cataract surgery, ${ }^{6-13} 4$ in pterygium surgery, ${ }^{14-17} 2$ in trabeculectomy/Ahmed valve implantation, ${ }^{18,19}$ and 1 on phaco/trabeculectomy combination procedures. ${ }^{20}$ There were 2 papers on lidocaine gel for intravitreal injection, ${ }^{21,22}$ and 1 on vitrectomy. ${ }^{23}$ In addition there was 1 paper each on lidocaine gel in the setting of penetrating keratoplasty, ${ }^{24}$ strabismus surgery, ${ }^{25}$ and chalazion excision. $^{26}$

\section{Randomized controlled trials (RCT)}

Salient points from the 15 prospective randomized controlled trials are summarized in Table 1. In each of these RCTs, lidocaine gel $2 \%$ was compared to topical tetracaine drops, subconjunctival injection, retrobulbar injection, sham gel, or another anesthetic modality. Clinical endpoints included patient-reported pain scores on a visual analog scale or verbal pain score during anesthetic instillation, 


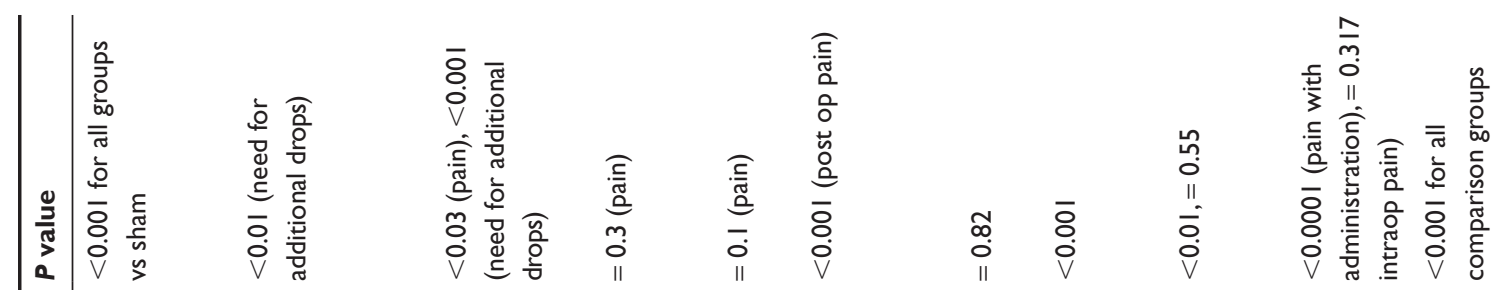
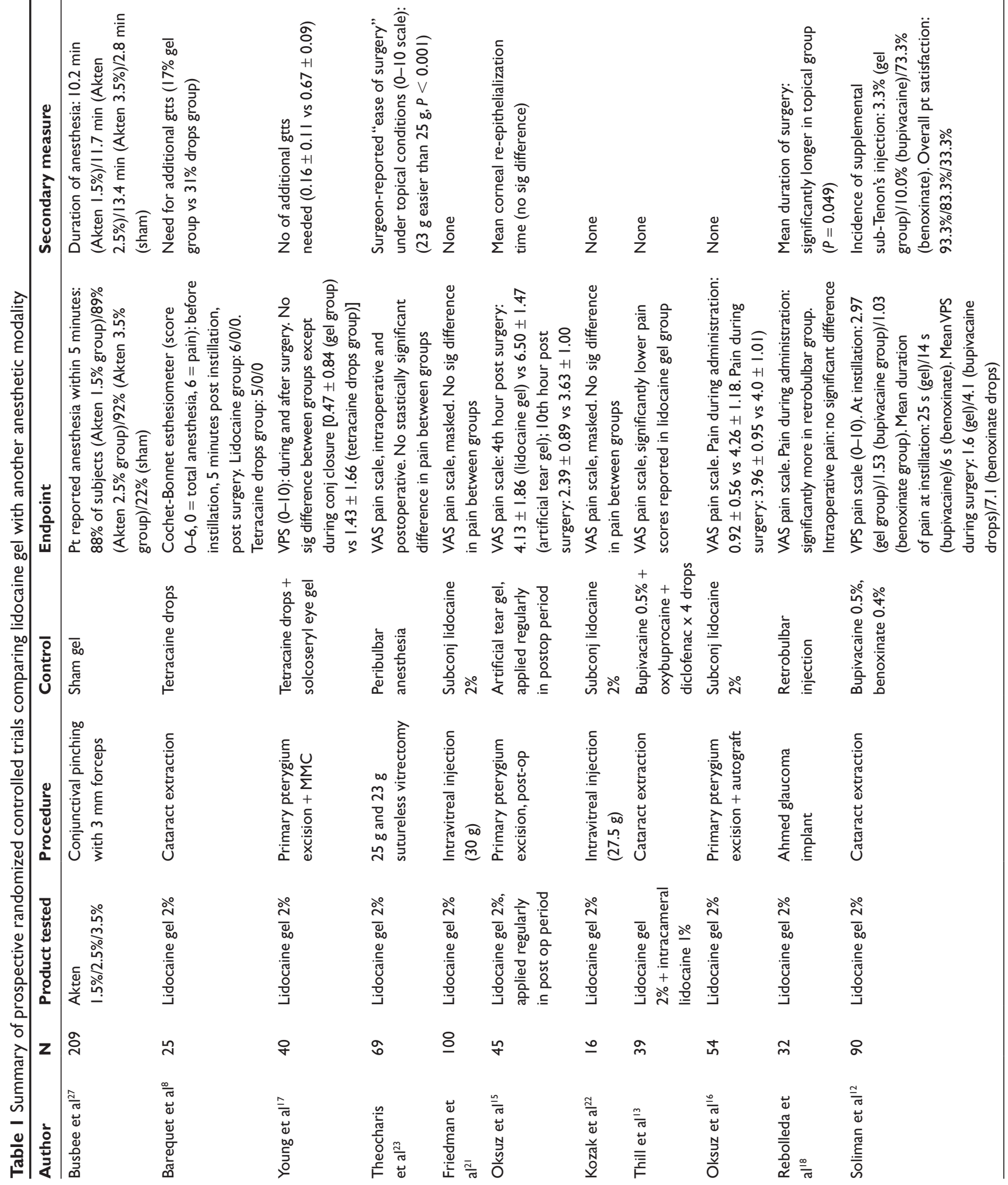


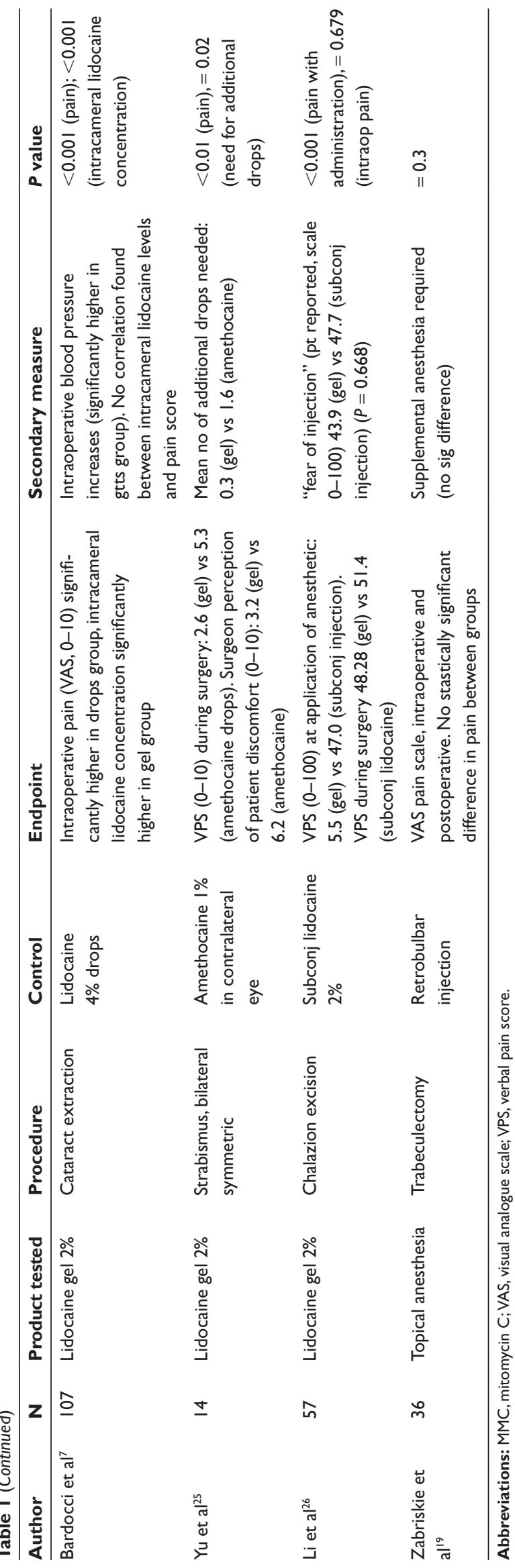

pain during surgery, and/or pain in the postoperative period. Other primary clinical endpoints included time to onset of anesthesia and duration of anesthesia. Secondary measures included subjective patient satisfaction, subjective surgeon satisfaction, need for additional anesthetic, and changes in intraoperative vital signs. The $15 \mathrm{RCTs}$ ranged in size from 14 to 209 subjects. A total of 933 patients were included in these 15 studies, with an average $\mathrm{N}$ of 62 . Two of the RCTs were double-blinded; $;^{7,27}$ the remaining 13 were unblinded but considered to be high-quality with the exception of 2 that were possibly underpowered $(\mathrm{N}<20) \cdot{ }^{22,25}$ Meta-analysis was not carried out on the studies due to their variable endpoints, differing surgical/procedural environments, and variable control groups across the studies.

\section{Subjective pain}

All 13 RCTs which measured intraoperative or postoperative pain with lidocaine gel vs another anesthetic modality relied upon patient reported pain levels, using typical verbal pain score (VPS, 0 to 10 , with 0 being no discomfort and 10 being excruciating pain) or visual analog scale (VAS, 0 to 10) measures. Eight out of 13 RCTs reported no significant difference in pain scores between the lidocaine gel group and the corresponding control group. Five out of 13 of these studies indicated a statistically significantly lower pain score in the lidocaine gel group compared with the control group ( $P$ values $<0.001$ to $<0.01$ ). Three studies reported a statistically significantly lower pain level at the time of administration of lidocaine gel vs subconjunctival lidocaine or retrobulbar block for Ahmed valve implantation (Rebollada et al $P<0.001$ ), ${ }^{18}$ pterygium excision (Oksuz et al $P<0.01$ ), ${ }^{16}$ or chalazion excision (Li et al $\left.P<0.001\right) .{ }^{26}$ One study (Soliman et al) ${ }^{12}$ found that patients reported a statistically significantly higher pain level at the time of administration of lidocaine gel vs administration of bupivicaine drops or benoxinate drops before cataract surgery $(P<0.001)$.

\section{Time to onset and duration of anesthesia}

Busbee et $\mathrm{al}^{27}$ showed that $88 \%$ to $92 \%$ of patients became anesthetic to conjunctival pinching with varying concentrations $(1.5 \%, 2.5 \%$, and $3.5 \%)$ of lidocaine gel within 5 minutes, while $22 \%$ of patients receiving sham gel also reported anesthesia to conjunctival pinching. In this study there was a dose-dependent variation in duration of anesthesia, with lidocaine gel $3.5 \%$ producing the longest duration of anesthesia $(P<0.001)$. Barequet et al ${ }^{8}$ demonstrated that both lidocaine gel and tetracaine drops 
produced equally adequate anesthesia (as measured with a Cochet-Bonnet anesthesiometer) at 5 minutes post-instillation and also when measured postoperatively.

\section{Need for additional anesthetic}

Five RCTs compared lidocaine gel with control groups and reported the total number of additional anesthetic applications required for patient comfort during cataract extraction, ${ }^{8,12}$ pterygium excision, ${ }^{17}$ strabismus surgery, ${ }^{25}$ and trabeculectomy. ${ }^{19}$ Four of these 5 studies demonstrated that the use of lidocaine gel as the primary preoperative anesthetic resulted in a statistically significantly lower number of supplemental anesthetic applications required during surgery to maintain patient comfort $(P<0.001$ to $<0.01)$. One of the studies showed no significant difference (lidocaine gel vs retrobulbar block for trabeculectomy). ${ }^{19}$

\section{Prospective nonrandomized trials}

The 6 nonrandomized prospective trials evaluating the use of lidocaine gel for ocular surgery ranged in size from 15 to 100 subjects, with an average $\mathrm{N}$ of 39 . These studies evaluated similar primary and secondary outcome measures as the randomized controlled trials, including intraoperative/ postoperative pain score, and need for additional anesthetic applications. See Table 2 for detailed information.

\section{Patient and surgeon satisfaction}

Several nonrandomized and randomized prospective trials tracked patient and surgeon satisfaction with the surgical experience under lidocaine gel anesthesia. Data were collected via subjective report and numeric scales. In a nonrandomized study of 15 patients undergoing repeat penetrating keratoplasty under topical lidocaine gel anesthesia (plus intracameral lidocaine and IV sedation) Segev et al reported that on a scale of 0 to $5(0=$ not at all satisfied, $5=$ very satisfied), average patient satisfaction was 4.67 and average surgeon satisfaction was 4.67. They report that $14 / 15$ patients preferred their surgical experience with topical lidocaine to their previous experience with retrobulbar injection..$^{24}$ In their RCT studying lidocaine gel use in cataract surgery, Soliman et al indicate that $93.3 \%$ of patients reported they were "satisfied" with their surgical experience under lidocaine gel anesthesia, compared with $83.3 \%$ of patients who received bupivacaine $0.5 \%$ drops and $33.3 \%$ of patients who received benoxinate $0.4 \%$ drops. ${ }^{12}$ In a series of 100 cataract extractions performed with lidocaine gel anesthesia, Assia et al noted improved lubrication of surgical instruments and easier entry and exit through surgical wounds. ${ }^{6}$
Finally, in his letter to the editor of Journal of Cataract and Refractive Surgery, Kirber noted excellent personal results in a series of cataract extractions performed with a 50/50 dilution of lidocaine $2 \%$ with balanced salt solution, including potent patient anesthesia and a similar improvement in instrument facility/lubrication. ${ }^{9}$

\section{Intracameral lidocaine levels}

In a nonrandomized prospective study of cataract surgery patients, Kwok et al demonstrated a strong correlation between duration of contact of lidocaine gel with the ocular surface and intracameral lidocaine levels measured by high performance liquid chromatography. However, they did not show any statistically significant correlation between intracameral lidocaine levels and pain score. ${ }^{10}$ In their RCT (also studying cataract surgery patients), Bardocci et al demonstrated that patients who received lidocaine gel $2 \%$ had significantly higher intracameral lidocaine levels than those receiving lidocaine $4 \%$ drops. This study similarly found no significant correlation between intracameral lidocaine levels and VPS pain score. ${ }^{7}$

\section{Adverse effects and safety}

The prospective randomized controlled trial by Busbee et al (examining timing of onset and duration of anesthesia with varying concentrations of lidocaine gel) specifically defined "adverse events" as corneal staining, conjunctival hyperemia, and/or pain with administration. They found the adverse event rate as defined here to be $2 \%$ to $6 \%$, comparable across all 209 subjects and across all treatment groups (lidocaine gel $1.5 \%, 2.5 \%, 3.5 \%$, and sham gel). ${ }^{27}$ The remainder of the human prospective trials reported an adverse event rate that was similarly low and not significantly different from controls or commonly reported side effects with topical anesthetic drops. Kozak et al demonstrated a statistically significantly higher rate of subconjunctival hemorrhage and chemosis among patients receiving subconjunctival lidocaine vs those receiving topical lidocaine gel prior to intravitreal injection $(P<0.001)$, with no difference in pain scores between the two groups $(P=0.82) .{ }^{22}$ Likewise, Zabriskie et al reported no difference in pain scores but a significantly higher rate of chemosis, subconjunctival hemorrhage, and eyelid hemorrhage in patients receiving retrobulbar injection vs lidocaine gel for trabeculectomy $(P<0.03){ }^{19}$

\section{Tissue toxicity}

In a rabbit study, Barequet et al demonstrated that neither topical lidocaine gel placed in the fornices of rabbit eyes nor 


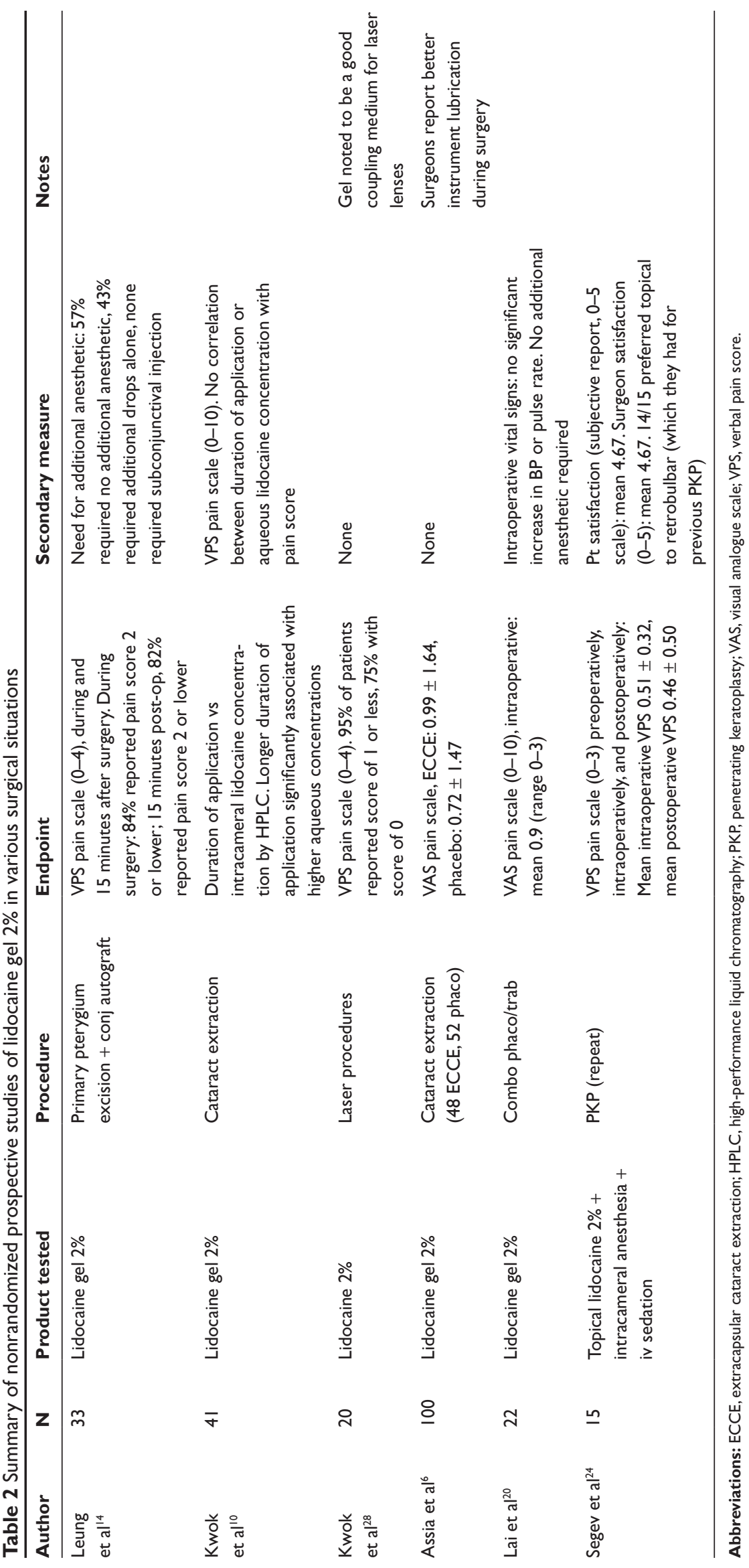


small volumes of lidocaine gel injected directly into the anterior chamber produced any significant clinical or histopathological alteration of ocular tissues. ${ }^{8}$ Schellini et al performed a series of anterior chamber injections into rabbit eyes with lidocaine gel $2 \%$ with preservative, lidocaine gel without preservative, preservative alone, and saline alone. They showed no significant difference across the groups in clinical, ultrastructural, or morphometric evaluations of the rabbit corneas, and no significant histological effects on corneal endothelial cells. ${ }^{29}$

\section{Effects on preoperative antisepsis}

Boden et al performed a microbiologic study on the interaction between lidocaine gel and povidone iodine, the most commonly used preoperative ocular antiseptic in the US. The authors inoculated sets of plates of blood agar with Staphylococcus aureus, Staphylococcus epidermidis, Pseudomonas aeruginosa, and Haemophilus influenza, and applied either lidocaine gel $2 \%$ alone, lidocaine gel plus povidone iodine $5 \%$, or povidone iodine alone to each set of plates. They reserved a set of plates for each organism with inoculum alone as a control. The plates were then cultured under standard conditions. The authors demonstrated that the number of colony forming units (CFUs) was similar in the control group (inoculum only), the lidocaineonly group, and the lidocaine + povidone group for S. aureus, $S$. epidermidis, and P. aeruginosa. Each set of plates in these groups grew between 200 and $300 \mathrm{CFUs}$, while the povidone + inoculum group for all 4 organisms grew only 0 to 6 CFUs. ${ }^{30}$ These results imply a decreased antimicrobial effectiveness of povidone iodine in the presence of lidocaine gel, which potentially could increase the risk for acute postoperative infection in ocular surgery performed under lidocaine gel anesthesia. On the clinical side, in a retrospective review of 15,920 cataract extractions performed at a single institution, Miller et al reported an incidence of 7/15,920 cases of acute postoperative endophthalmitis $(0.04 \%)$. This rate is consistent with other large reports in the literature, and is notable for the fact that 2 out of the 7 cases were performed under lidocaine gel topical anesthesia. ${ }^{11}$ The paper by Miller et al has been referenced numerous times in discussions that lidocaine gel may increase the risk of acute postoperative endophthalmitis, but there has been no other large scale study or statistically significant data published to date which establishes a higher rate of acute postoperative endophthalmitis under gel anesthesia versus other anesthetic modalities.

\section{Conclusions}

Thanks to the work of pioneers throughout the 19th and 20th centuries, ophthalmic surgeons today have a wide variety of anesthetic tools at their disposal. Depending on the surgical procedure to be performed, patient characteristics, and surgeon factors, the preferred anesthetic modality may include general anesthesia, iv sedation, retrobulbar or peribulbar blockade, topical anesthesia, or any combination thereof. There will always be cases where general anesthesia confers extra safety (pediatric, mentally retarded, and demented populations to name a few examples). Likewise there are some scenarios where total akinesia and anesthesia is paramount and retrobulbar injection provides the safest and most stable surgical environment.

With advances in surgical techniques and instrumentation, there has been a general trend toward smaller incisions and less invasive maneuvers in ophthalmic surgery. This has been accompanied over the last 25 years by a renewed interest in topical anesthetic techniques, which in many cases enhance patient comfort and reduce the inherent risks of retrobulbar and peribulbar injection. These risks include but are not limited to ocular penetration and perforation (approximately 1 in 1000), retrobulbar hemorrhage (1\% to $3 \%$ ), central nervous depression ( 1 per 350 to 500 cases) ${ }^{33}$ and pain with injection requiring substantial premedication by the anesthesiologist.

The series of papers presented here indicate that lidocaine gel is at least as effective and often more effective than widely available topical anesthetic drops for treating and preventing cataract surgery-related pain. Where lidocaine gel is compared to retrobulbar and subconjunctival anesthesia for a variety of non-cataract procedures (pterygia, glaucoma surgery, strabismus, vitreoretinal surgery, and corneal transplant), pain control has been shown to be at least as good, and with fewer adverse effects. There have been a limited number of studies in these areas, however, and with relatively small groups of patients. The 15 randomized controlled trials discussed here were determined to be of moderate to high quality, with some being underpowered. The 6 nonrandomized prospective trials do not offer additional statistical support for the use of lidocaine gel but the data provided is fairly convincing of the product's utility.

Patient and surgeon satisfaction with the use of lidocaine gel has been high, with patients reporting improved comfort and several practitioners noting improved instrument lubrication and facility of surgery due to the gel's physical characteristics.

A handful of animal studies have been carried out to demonstrate the safety of lidocaine gel and its lack of significant clinical or histopathological adverse effects on ocular tissues. On the other hand, questions have been raised as to the reduced 
efficacy of povidone iodine antisepsis when applied after lidocaine gel as part of the preoperative regimen, which could potentially produce a substantial increase in risk of acute postoperative infection. In our search of the literature we found one large case series (Miller et al) ${ }^{11}$ which suggests an increased risk of acute postoperative endophthalmitis with lidocaine gel anesthesia, but the overall incidence is very low $(0.04 \%)$ and it is difficult to draw any statistically significant conclusions about the role of the anesthetic. The paper by Miller has been referenced numerous times in discussions on lidocaine gel and endophthalmitis risk, but to our knowledge there has been no other large case series or presentation of statistically significant data (other than the microbiological study discussed here) which indicates a real increase in the risk. Furthermore, until recently the majority of lidocaine gel used for ophthalmic surgery was not originally manufactured for this use and did not come packaged in sterile dropper form (which may have increased the risk of contamination). Now that the gel formulations are available in sterile packaging and in a format more suitable for ophthalmic application, the risk of postoperative infection may be mitigated even further. Other means of decreasing the risk of contamination and increasing the effectiveness of povidone iodine antisepsis could include the copious irrigation of the conjunctival fornices after adequate preoperative contact with the gel and prior to povidone instillation.

In the overall, lidocaine gel appears to be a safe and highly efficacious tool for ocular anesthesia, with high patient and surgeon satisfaction when the product is employed appropriately. Based upon our findings in the literature we would support its use. More data are needed on lidocaine gel and acute postoperative endophthalmitis risk, as a perceived increase in the risk is likely the most significant deterrent to its acceptance and use by more ophthalmic surgeons.

\section{Acknowledgments}

This work was supported in part by an unrestricted grant to Casey Eye Institute from Research to Prevent Blindness, New York, NY.

\section{Disclosures}

Dr Fraunfelder serves as a consultant to Brymill. The authors have no proprietary interest in the materials discussed herein.

\section{References}

1. Koller K. On the use of cocaine to anesthetize the eye. As translated by Knapp H. Arch Ophthalmol. 1884;13:402-448.

2. Knapp H. On cocaine and its use in ophthalmic and general surgery. Arch Ophthalmolol. 1884;13:402.
3. Gills JP, Hustead RF, Sanders DR. Ophthalmic Anesthesia. New York, NY: McGraw-Hill; 1993.

4. Atkinson WS. Larger volume retrobulbar injections. Am J Ophthalmol. 1948;31:1607-1618.

5. Marr WG, Wood R, Senterfit L, Sigelman S. Effect of topical anesthetics on regeneration of corneal epithelium. Am J Ophthalmol. 1957;43(4 Pt 1):606-610.

6. Assia EI, Pras E, Yehezkel M, Rotenstreich Y, Jager-Roshu S. Topical anesthesia using lidocaine gel for cataract surgery. $J$ Cataract Refract Surg. 1999;25(5):635-639.

7. Bardocci A, Lofoco G, Perdicaro S, Ciucci F, Manna L. Lidocaine $2 \%$ gel versus lidocaine $4 \%$ unpreserved drops for topical anesthesia in cataract surgery: a randomized controlled trial. Ophthalmology. 2003;110(1):144-149.

8. Barequet IS, Soriano ES, Green WR, O'Brien TP. Provision of anesthesia with a single application of lidocaine $2 \%$ gel. $J$ Cataract Refract Surg. 1999;25(5):626-631.

9. Kirber WM. Lidocaine gel for topical anesthesia. $J$ Cataract Refract Surg. 2000 Feb;26(2):163.

10. Kwok AK, Lai TY, Lee VY, Yeung YS, Chu KO, Pang CC. Effect of application duration of $2 \%$ lidocaine jelly on aqueous lidocaine concentration for topical anesthesia in cataract surgery. Graefes Arch Clin Exp Ophthalmol. 2006;244(9):1096-1100.

11. Miller JJ, Scott IU, Flynn HW Jr, Smiddy WE, Newton J, Miller D. Acute-onset endophthalmitis after cataract surgery (2000-2004): incidence, clinical settings, and visual acuity outcomes after treatment. Am J Ophthalmol. 2005;139(6):983-987.

12. Soliman MM, Macky TA, Samir MK. Comparative clinical trial of topical anesthetic agents in cataract surgery: lidocaine $2 \%$ gel, bupivacaine $0.5 \%$ drops, and benoxinate $0.4 \%$ drops. J Cataract Refract Surg. 2004;30(8):1716-1720.

13. Thill M, Zeitz O, Richard I, Richard G. Lidocaine gel versus combined topical anesthesia using bupivacaine, oxybuprocaine and diclofenac eyedrops in cataract surgery. Ophthalmologica. 2005;219(3): 167-170.

14. Leung GY, Young AL, Wong AK, Rao SK, Lam DS. Efficacy of $2 \%$ lignocaine gel in pterygium surgery. Acta Ophthalmol Scand. 2006;84(3):445

15. Oksuz H, Tamer C. Pain relief after pterygium surgery with viscous lidocaine. Ophthalmologica. 2006;220(5):323-326.

16. Oksuz H, Tamer C. Efficacy of lidocaine $2 \%$ gel in pterygium surgery. Acta Ophthalmol Scand. 2005;83(2):206-209.

17. Young AL, Leung GY, Cheng LL, Lau TT, Lam PT, Lam DS. Randomised controlled trial on the effectiveness of lidocaine gel vs tetracaine drops as the sole topical anaesthetic agent for primary pterygium surgery. Eye. 2009;23(7):1518-1523.

18. Rebolleda G, Muñoz-Negrete FJ, Gutierrez-Ortiz C. Topical plus intracameral lidocaine versus retrobulbar anesthesia in phacotrabeculectomy: prospective randomized study. J Cataract Refract Surg. 2001;27(8):1214-1220.

19. Zabriskie NA, Ahmed II, Crandall AS, Daines B, Burns TA, Patel BC. A comparison of topical and retrobulbar anesthesia for trabeculectomy. J Glaucoma. 2002;11(4):306-314.

20. Lai JS, Tham CC, Lam DS. Topical anesthesia in phacotrabeculectomy. J Glaucoma. 2002;11(3):271-274.

21. Friedman SM, Margo CE. Topical gel vs subconjunctival lidocaine for intravitreous injection: a randomized clinical trial. Am J Ophthalmol. 2006;142(5):887-888.

22. Kozak I, Cheng L, Freeman WR. Lidocaine gel anesthesia for intravitreal drug administration. Retina. 2005;25(8):994-998.

23. Theocharis IP, Alexandridou A, Tomic Z. A two-year prospective study comparing lidocaine $2 \%$ jelly versus peribulbar anaesthesia for $25 \mathrm{G}$ and 23G sutureless vitrectomy. Graefes Arch Clin Exp Ophthalmol. 2007;245(9):1253-1258.

24. Segev F, Voineskos AN, Hui G, et al. Combined topical and intracameral anesthesia in penetrating keratoplasty. Cornea. 2004; 23(4):372-376. 
25. Yu CB, Wong VW, Fan DS, Yip WW, Lam DS. Comparison of lidocaine $2 \%$ gel versus amethocaine as the sole anesthetic agent for strabismus surgery. Ophthalmology. 2003;110(7):1426-1429.

26. Li RT, Lai JS, Ng JS, Law RW, Lau EM, Lam DS. Efficacy of lignocaine 2\% gel in chalazion surgery. Br J Ophthalmol. 2003;87(2):157-159.

27. Busbee BG, Alam A, Reichel E. Lidocaine hydrochloride gel for ocular anesthesia: results of a prospective, randomized study. Ophthalmic Surg Lasers Imaging. 2008;39(5):386-390.

28. Kwok AK, Young AL, Lam DS. Efficacy of lignocaine gel for outpatient laser treatment in inflamed eyes. Eye. 2001;15(Pt 5):608-611.

29. Schellini SA, Creppe MC, Gregorio EA, Padovani CR. Lidocaine effects on corneal endothelial cell ultrastructure. Vet Ophthalmol. 2007;10(4):239-244.
30. Boden JH, Myers ML, Lee T, Bushley DM, Torres MF. Effect of lidocaine gel on povidone-iodine antisepsis and microbial survival. J Cataract Refract Surg. 2008;34(10):1773-1775.

31. Rebolleda G, Muñoz-Negrete FJ, Benatar J, Corcostegui J, Alonso N. Comparison of lidocaine $2 \%$ gel versus retrobulbar anaesthesia for implantation of Ahmed glaucoma drainage. Acta Ophthalmol Scand. 2005;83(2):201-205.

32. Gaynes BI. Lidocaine gel versus drops. Ophthalmology. 2003;110(12) 2429-2430; Author reply 2430.

33. Schecter RJ. Management of inadvertent intraocular injections. Ann Ophthalmol. 1985;17:771-175.
Clinical Ophthalmology

\section{Publish your work in this journal}

Clinical Ophthalmology is an international, peer-reviewed journal covering all subspecialties within ophthalmology. Key topics include: Optometry; Visual science; Pharmacology and drug therapy in eye diseases; Basic Sciences; Primary and Secondary eye care; Patien Safety and Quality of Care Improvements. This journal is indexed on

Submit your manuscript here: http://www.dovepress.com/clinical-ophthalmology-journal

\section{Dovepress}

PubMed Central and CAS, and is the official journal of The Society of Clinical Ophthalmology (SCO). The manuscript management system is completely online and includes a very quick and fair peer-review system, which is all easy to use. Visit http://www.dovepress.com/ testimonials.php to read real quotes from published authors. 